\title{
EchoGéo
}

26 | 2013

Varia

\section{Les fleurs de Naivasha}

Jean-Louis Chaléard

\section{OpenEdition}

Journals

Édition électronique

URL : https://journals.openedition.org/echogeo/13674

DOI : 10.4000/echogeo. 13674

ISSN : 1963-1197

\section{Éditeur}

Pôle de recherche pour l'organisation et la diffusion de l'information géographique (CNRS UMR 8586)

Référence électronique

Jean-Louis Chaléard, « Les fleurs de Naivasha », EchoGéo [En ligne], 26 | 2013, mis en ligne le 19 décembre 2013, consulté le 31 juillet 2021. URL : http://journals.openedition.org/echogeo/13674 ; DOI : https://doi.org/10.4000/echogeo.13674

Ce document a été généré automatiquement le 31 juillet 2021.

EchoGéo est mis à disposition selon les termes de la licence Creative Commons Attribution - Pas d'Utilisation Commerciale - Pas de Modification 4.0 International (CC BY-NC-ND) 


\title{
Les fleurs de Naivasha
}

\author{
Jean-Louis Chaléard
}

1 La nouvelle livraison d'ÉchoGéo est fournie, témoignant de l'intérêt de nombreux auteurs pour la formule « varia » adoptée depuis 2011 par la revue. Il serait certes vain de vouloir trouver une unité à ce numéro, par définition placé sous le signe de la diversité. Chemin faisant apparaissent cependant quelques tendances ou récurrences qui manifestent des focalisations sur des thèmes sensibles aux géographes.

2 Trois articles sont consacrés, sous des formes diverses, à des questions nouvelles qui touchent à l'urbain, sans être focalisé directement dessus: mutations des espaces urbanisés, influence des métropoles, relations inédites entre les centres et leurs périphéries, évolution des manières de vivre et de voir la ville comme les campagnes. Le texte sur les services hospitaliers dans l'agglomération de Sfax montre à la fois le pouvoir d'attraction du centre-ville dans une agglomération d'un pays du Sud, mais aussi les problèmes de congestion qui en découlent. La contribution sur le tourisme rural en Chine traite de beaucoup plus que de questions sur les seules campagnes. D'abord, il donne à voir une facette nouvelle du pays, avec l'essor du tourisme et des loisirs. Ensuite il révèle un visage singulier des périphéries métropolitaines dont les dynamiques sont liées au centre urbain. Car c'est du tourisme à la périphérie de Shanghai, par et pour les Shanghaiens, dont il s'agit, mettant en évidence la diversification des activités autour d'une grande métropole. Le texte sur la patrimonialisation et les greffes culturelles sur les friches issues de l'industrie minière, à partir des exemples des bassins miniers de la vallée de l'Emscher (dans la Ruhr allemande) et du Nord-Pas de Calais (en France), analyse les modalités de la transformation des infrastructures minières en lieux culturels, renvoyant à des thèmes concernant l'aménagement urbain, la créativité, la culture, qui contribuent aussi à changer la vie quotidienne et les représentations sur la ville.

3 Un thème fort que l'on peut sans doute aussi relever dans ce numéro est celui qui concerne l'environnement. Bien sûr on le trouve dans toutes les analyses ayant trait à la ville durable, au «besoin " d'espaces verts des citadins et au tourisme rural à la périphérie des métropoles qui en est une manifestation. L'environnement est abordé de façon directe dans trois communications, portant sur des aspects différents mais qui, à 
des degrés divers, mettent en cause l'action des hommes. Le premier article traite des risques dans l'intérieur du Nordeste brésilien, à partir de la crue-éclair du Rio Grangeiro du 28 janvier 2011. Il met en évidence les facteurs naturels et anthropiques en cause dans ce type de catastrophe, soulignant le rôle d'une urbanisation mal contrôlée qui modifie les modalités d'écoulement et aggrave les menaces sur les biens et les personnes. Le deuxième article aborde les conséquences physiques des prélèvements de sables et graviers dans la basse vallée du Mékong, de plus en plus élevés avec l'essor économique des pays traversés par le fleuve, produisant des changements géomorphologiques et écologiques importants dans le delta. D'environnement il est encore question dans la contribution sur les enjeux et conséquences de la réglementation sur le bois-énergie au Tchad. Ici comme dans beaucoup de pays du Sahel la déforestation est un thème d'actualité et au cœur des politiques publiques concernant l'environnement. L'article montre comment ces politiques répondent plus à la volonté d'affirmer l'autorité du pouvoir central au lendemain d'une profonde crise politico-militaire et de capter de nouvelles rentes environnementale mondialisées, qu'à un désir d'apporter de véritables solutions durables.

4 Le texte sur la mondialisation, la clusterisation et le recyclage colonial est à la fois singulier et contient des thèmes que l'on retrouve dans les autres textes: métropolisation, influences venues d'un monde globalisé, problèmes environnementaux issus d'activités mal contrôlées... Il analyse la production de roses dans la région de Naivasha, près de Nairobi au Kenya, montrant comment la mise en valeur coloniale, les reconversions postcoloniales, les avantages comparatifs dans un système d'échange mondialisé ont permis la mise en place d'un «Système Productif Localisé » original.

5 Le propos de la rubrique Sur l'Écrit n'est pas très éloigné des questions abordées dans plusieurs articles et qui ont trait à la mondialisation, notamment celui sur la production de roses au Kenya qui aborde aussi les conditions de travail des ouvriers. L'entretien entre Albena Tcholakova et Djemila Zeneidi, à travers l'étude des saisonnières agricoles marocaines en Espagne, nous conduit au cœur de nouveaux espaces productifs où domination et émancipation se conjuguent de manière ambivalente. Les analyses, dans l'ouvrage dont il est question, mobilisent les apports d'auteurs variés, de Foucault à Harvey, dans une approche originale, pour rendre compte de la complexité du cheminement de ces femmes, où se mêlent surveillance stricte au travail, cadre moral rigide du milieu d'origine et désir d'autonomie.

6 La rubrique Sur le Métier est consacrée à la croissance des études sur la religion dans les sciences sociales et à l'usage des sciences sociales par les religions. La présentation de Frédéric Dejean, comme l'interview de Glenn Smith, professeur de théologie et spécialiste de la ville, illustrent la dimension géographique des études sur la religion et la complexité des usages que les chrétiens évangéliques peuvent faire des recherches urbaines puisqu'elles se trouvent intégrées au cœur du travail théologique et missionnaire. La géographie ne sert pas qu'à faire la guerre...

7 Le nouvel article sur Chypre, dans la rubrique Sur le Vif, met en évidence les ambiguïtés de la situation et les problèmes posés à l'Union européenne par la division de l'île et l'occupation turque. Ce texte aurait pu s'inscrire dans la livraison précédente consacrée au Moyen-Orient, car l'île est au milieu d'un bassin méditerranéen oriental en pleine ébullition. Mais la République de Chypre fait partie de l'Union européenne, même si elle 
en est une périphérie instable. Et la découverte de gisements de gaz dans la région comme les évènements récents en Afrique du Nord-Est et en Asie Occidentale changent en partie la donne et les points de vue à Bruxelles, ouvrant la porte à des évolutions nouvelles.

8 On peut constater que la plupart des textes de la nouvelle livraison d'ÉchoGéo abordent des cas étrangers. Cela tient sans doute à l'orientation de la revue très ouverte sur le monde. On peut penser aussi que l'analyse des périphéries, des marges spatiales et sociales, ou des pays du Sud en proie à des problèmes de surexploitation des ressources, de pauvreté, d'urbanisation mal contrôlée est révélatrice de problèmes au cœur des sociétés les plus fortunées, des modèles de développement dominants, des conséquences de l'accumulation des richesses dans de grandes métropoles. Du bassin minier du Nord en proie à la reconversion au tourisme à la périphérie de Shanghai ou aux fleurs du Kenya, tous ces cas mettent en évidence des dynamiques nouvelles et des problèmes actuels auxquels est confronté le monde contemporain. 